PROCEEDINGS OF THE

AMERICAN MATHEMATICAL SOCIETY

Volume 123, Number 4, April 1995

\title{
LINEAR MAPPINGS THAT PRESERVE POTENT OPERATORS
}

\author{
MATJAŽ OMLADIČ AND PETER ŠEMRL
}

(Communicated by Palle E. T. Jorgensen)

\begin{abstract}
Let $H$ and $K$ be a complex Hilbert spaces, while $\mathscr{B}(H)$ and $\mathscr{B}(K)$ denote the algebras of all linear bounded operators on $H$ and $K$, respectively. We characterize surjective linear mappings from $\mathscr{B}(H)$ onto $\mathscr{B}(K)$ that preserve potent operators in both directions.
\end{abstract}

The problem of characterizing linear mappings $\phi$ on the algebra $M_{n}$ of all $n \times n$ matrices which preserve some subsets $\Gamma$ of $M_{n}$ (that is, $\left.\phi(\Gamma) \subset \Gamma\right)$ has attracted the attention of many mathematicians in the last few decades [12]. Let us mention here some examples of such subsets: the case when $\Gamma$ is the set of all singular matrices was considered by Dieudonné [7], $\Gamma$ is a linear group by Dixon [8], and $\Gamma$ is the set of all nilpotent matrices by Botta, Pierce, and Watkins [1]. In a recent paper [2], motivated by a problem of characterizing local automorphisms and local derivations of some operator algebras (see, e.g., [11]), Brešar and Šemrl considered the case when $\Gamma$ is the set of all idempotents in $M_{n}$. The same authors also considered a more general situation [3], namely, they characterized linear transformations preserving potent matrices (recall that a matrix $A$ is said to be potent if $A^{r}=A$ for some integer $r \geq 2$ ) as well as linear mappings that preserve the set of all $r$-potent matrices $\Pi_{r}=\left\{A \in M_{n}\right.$ : $\left.A^{r}=A\right\}$ for some integer $r \geq 2$.

In recent years there has also been considerable interest in linear preserver problems on operator algebras over infinite-dimensional spaces $[2,4,5,6,9,13$, 14]. It is the aim of this note to continue this work by studying linear mappings $\phi: \mathscr{B}(H) \rightarrow \mathscr{B}(K)$ that preserve potent operators in both directions. Here, $H$ and $K$ are nontrivial complex Hilbert spaces, while $\mathscr{B}(H)$ and $\mathscr{B}(K)$ denote the algebras of all bounded linear operators on $H$ and $K$, respectively. The main idea is different from the one used in the finite-dimensional case [3]. We also need a stronger assumption on $\phi$ : it must preserve potent operators in both directions, that is, for every $A \in \mathscr{B}(H)$ the operator $\phi(A)$ is a potent operator if and only if the same is true for $A$.

Our proof is based on the following three results.

Theorem 1 [15]. Let $H$ be an infinite-dimensional Hilbert space. Then every operator $A \in \mathscr{B}(H)$ is a sum of five idempotents.

Received by the editors June 25, 1993.

1991 Mathematics Subject Classification. Primary 47B49.

This work was supported by a grant from the Ministry of Science of Slovenia. 
Theorem 2 [10]. Let $\mathscr{A}$ be a Banach algebra, and let $p, q \in \mathscr{A}$ be idempotents. Then $p+q$ is an idempotent if and only if $\sup _{n \in \mathbb{N}}\left\|(p+q)^{n}\right\|<\infty$.

Theorem 3 [4]. Let $H$ and $K$ be Hilbert spaces, and let $\phi: \mathscr{B}(H) \rightarrow \mathscr{B}(K)$ be a bijective linear mapping. Assume that $\phi(P) \in \mathscr{B}(K)$ is an idempotent whenever $P \in \mathscr{B}(H)$ is an idempotent. Then there is a bounded bijective linear operator $V: H \rightarrow K$ such that either

(i) $\phi(A)=V A V^{-1}$ for every $A \in \mathscr{B}(H)$; or

(ii) $\phi(A)=V A^{\text {tr }} V^{-1}$ for every $A \in \mathscr{B}(H)$, where $A^{\text {tr }}$ denotes the transpose of $A$ relative to a fixed but arbitrary orthonormal basis.

This last result was proved in [4] only for the special case $H=K$. Almost the same proof works also in this more general setting.

Throughout the paper, for any vectors $x, y$ we shall denote the scalar product of these two vectors by $y^{*} x$, while $x y^{*}$ will denote the rank-one operator defined by $\left(x y^{*}\right) z=\left(y^{*} z\right) x$ for every vector $z$. Note that every operator of rank one can be written in this form. The operator $x y^{*}$ is an idempotent if and only if $y^{*} x=1$. Now we are ready to prove our result.

Theorem 4. Let $H$ and $K$ be Hilbert spaces, and let $\phi: \mathscr{B}(H) \rightarrow \mathscr{B}(K)$ be a surjective linear mapping. Then the following conditions are equivalent:

(i) For every $A \in \mathscr{B}(H)$ the operator $\phi(A)$ is potent if and only if $A$ is potent.

(ii) There exists an integer $r \geq 2$ such that for every $A \in \mathscr{B}(H)$ we have $(\phi(A))^{r}=\phi(A)$ if and only if $A^{r}=A$.

(iii) $\phi$ is either of the form $\phi(A)=c V A V^{-1}$ or $\phi(A)=c V A^{\text {tr }} V^{-1}$, where $V: H \rightarrow K$ is a bounded bijective linear operator, $c \in \mathbb{C}$ is a root of unity, and $A^{\text {tr }}$ denotes the transpose of $A$ relative to any basis of $H$, fixed in advance.

Proof. It is clear that (iii) implies (i) and (ii). We shall prove the converse implications. Let us first point out a simple observation which will be used later. An operator $A \in \mathscr{B}(H)$ is $r$-potent if and only if there exist nonzero idempotents $P_{1}, \ldots, P_{k}$ and pairwise different $(r-1)$-roots of unity $\mu_{1}, \ldots, \mu_{k}$ such that $A=\sum_{i=1}^{k} \mu_{i} P_{i}$ and $P_{i} P_{j}=0$ for $i \neq j$. In order to see this we denote

$$
\lambda_{0}=0 \quad \text { and } \quad \lambda_{m}=\exp \left(\frac{2 m \pi i}{r-1}\right), \quad m=1, \ldots, r-1 .
$$

The polynomials $p_{0}, p_{1}, \ldots, p_{r-1}$ defined by relations

$$
p_{i}(\lambda)=\prod_{m=0, m \neq i}^{r-1}\left(\lambda-\lambda_{m}\right), \quad \text { for } i=0,1, \ldots, r-1,
$$

have the greatest common divisor equal to 1 , so that there exist polynomials $q_{0}, \ldots, q_{r-1}$ with the property that $\sum_{i=0}^{r-1} p_{i} q_{i}=1$. It follows that every $x$ from $H$ can be written as $x=\sum_{i=0}^{r-1} x_{i}$ where $x_{i}=p_{i}(A) q_{i}(A) x, i=0, \ldots, r-$ 1. Clearly, we have that $x_{i} \in \operatorname{Ker}\left(A-\lambda_{i}\right), i=0,1, \ldots, r-1$. Thus, we have proved that

$$
H=\bigoplus_{i=0}^{r-1} H_{i}
$$


where $H_{i}=\operatorname{Ker}\left(A-\lambda_{i}\right), i=0,1, \ldots, r-1$. It is easy to see that the nontrivial among the projections $P_{i}$ of $H$ onto $H_{i}$ for $i=1, \ldots, r-1$, associated with the above direct sum, have the desired property.

Assume now that condition (i) is satisfied. Then the mapping $\phi$ is injective, since the kernel of $\phi$ is a linear space which consists of potent operators only. This implies that $\phi$ is actually bijective.

Clearly, operator $S=\phi(I)$ is potent so that, by above, there exist a positive integer $k$, nonzero idempotents $Q_{1}, \ldots, Q_{k} \in \mathscr{B}(K)$, and pairwise different roots of unity $\mu_{1}, \ldots, \mu_{k}$ such that $S=\sum_{i=1}^{k} \mu_{i} Q_{i}$ and $Q_{i} Q_{j}=0$ for $i \neq j$. We claim that $\sum_{i=1}^{k} Q_{i}=I$. Assume to the contrary that $Q=I-\sum_{i=1}^{k} Q_{i}$ is a nonzero idempotent. Then $S+c Q$ is a potent operator for every root of unity $c$. It follows that $\phi^{-1}(S+c Q)=I+c \phi^{-1}(Q)$ is a potent operator for every root of unity $c$, where $\phi^{-1}(Q) \neq 0$. This leads to a contradiction, thus showing that $\sum_{i=1}^{k} Q_{i}=I$.

Observe that $P_{i}=\mu_{i} \phi^{-1}\left(Q_{i}\right)$ are nonzero potents for $i=1, \ldots, k$. Clearly, $S-\mu_{i} Q_{i}$ and $S-2 \mu_{i} Q_{i}$ are all potent operators. The same must therefore be true for operators $I-P_{i}$ and $I-2 P_{i}$, and this implies that $P_{i}$ is an idempotent for every $i=1, \ldots, k$. Obviously, we have that $I=\sum_{i=1}^{k} P_{i}$. Moreover, $\mu_{i} Q_{i}+\mu_{j} Q_{j}$ is a potent operator if $i \neq j$. By the assumption, this implies that $P_{i}+P_{j}$ is a potent operator. Using Theorem 2 we conclude that $P_{i} P_{j}=0$ for $i \neq j$.

The algebra $\mathscr{A}_{i}=\left\{A \in \mathscr{B}(H): P_{i} A P_{i}=A\right\}, i=1, \ldots, k$, is isomorphic to $\mathscr{B}\left(H_{i}\right)$, where $H_{i}$ denotes the image of the idempotent $P_{i}$. Similarly, for every $i=1, \ldots, k$, the algebra $\mathscr{B}_{i}=\left\{A \in \mathscr{B}(K): Q_{i} A Q_{i}=A\right\}$ is isomorphic to $\mathscr{B}\left(K_{i}\right)$ with $K_{i}=\operatorname{Im} Q_{i}$. Fix $i$ for a while, and let $P$ be an arbitrary idempotent in $\mathscr{A}_{i}$. If we denote $T=\sum_{j=1}^{k} \mu_{j}^{-1} P_{j}$, then $T-\mu_{i}^{-1} P$ and $T-2 \mu_{i}^{-1} P$ are potent operators in $\mathscr{B}(H)$. It follows that $I-\mu_{i}^{-1} \phi(P)$ and $I-2 \mu_{i}^{-1} \phi(P)$ are potent operators, which further forces $\mu_{i}^{-1} \phi(P)$ to be an idempotent. Since $\mu_{i}^{-1} P+\mu_{j}^{-1} P_{j}$ is a potent operator for every $j$ different from $i$, the sum of idempotents $\mu_{i}^{-1} \phi(P)+Q_{j}$ is a potent operator, and consequently, by Theorem 2, we have that $\phi(P) Q_{j}=Q_{j} \phi(P)=0$, or in other words, $\phi(P)$ belongs to $\mathscr{B}_{i}$. The linear span of all idempotents from $\mathscr{A}_{i}$ is the whole algebra $\mathscr{A}_{i}$. One can easily verify this fact in the case that $H_{i}$ is finite dimensional, while in the infinite-dimensional case this statement follows from Theorem 1. This implies that $\phi$ maps $\mathscr{A}_{i}$ into $\mathscr{B}_{i}$. Similarly, we can prove that $\phi^{-1}$ maps $\mathscr{B}_{i}$ into $\mathscr{A}_{i}$, or in other words, the restriction of $\phi$ to the subalgebra $\mathscr{A}_{i}$ is a bijective mapping from $\mathscr{A}_{i}$ onto $\mathscr{B}_{i}$ for every $i=1, \ldots, k$. Applying Theorem 3 we see, in particular, that $\mu_{i}^{-1} \phi$ maps every idempotent of rank one from $\mathscr{A}_{i}$ into an idempotent of rank one from $\mathscr{B}_{i}$. This is true, of course, for any index $i=1,2, \ldots, k$.

Next, we shall prove that $k=1$ or, equivalently, that $\phi(I)=c I$ for some root of unity $c$. Assume to the contrary that $k>1$. Let $x y^{*} \in \mathscr{A}_{1}$ and $z w^{*} \in \mathscr{A}_{2}$ be any idempotents of rank one. Then $y^{*} x=w^{*} z=1$, and also, $\left(x y^{*}\right)\left(z w^{*}\right)=\left(z w^{*}\right)\left(x y^{*}\right)=0$, and this implies that $y^{*} z=w^{*} x=0$. Let us denote $\phi\left(x y^{*}\right)=\mu_{1} x_{1} y_{1}^{*}$ and $\phi\left(z w^{*}\right)=\mu_{2} z_{1} w_{1}^{*}$ to get that by the above $y_{1}^{*} x_{1}=w_{1}^{*} z_{1}=1$ and $y_{1}^{*} z_{1}=w_{1}^{*} x_{1}=0$.

For every complex number $\lambda$ the operators $x y^{*}+\lambda x w^{*}$ and $z w^{*}+\lambda x w^{*}$ 
are idempotents. It follows that for every complex number $\lambda$ there exists an integer $n_{\lambda} \geq 2$ such that

$$
\left(x_{1} y_{1}^{*}+\lambda \phi\left(x w^{*}\right)\right)^{n_{\lambda}}=x_{1} y_{1}^{*}+\lambda \phi\left(x w^{*}\right) .
$$

Clearly, there exists an integer $n_{0} \geq 2$ such that the above relation with $n_{0}=n_{\lambda}$ holds for infinitely many $\lambda$ 's, but then this must be fulfilled for every complex $\lambda$. Comparing the coefficients at $\lambda$ we obtain

$$
\phi\left(x w^{*}\right) x_{1} y_{1}^{*}+\left(n_{0}-2\right) x_{1} y_{1}^{*} \phi\left(x w^{*}\right) x_{1} y_{1}^{*}+x_{1} y_{1}^{*} \phi\left(x w^{*}\right)=\phi\left(x w^{*}\right) .
$$

Multiplying this relation from both sides by $x_{1} y_{1}^{*}$ we get that $x_{1} y_{1}^{*} \phi\left(x w^{*}\right) x_{1} y_{1}^{*}$ $=0$. Putting $u=\phi\left(x w^{*}\right) x_{1}$ and $v=\phi\left(x w^{*}\right)^{*} y_{1}$ we finally conclude that

$$
\phi\left(x w^{*}\right)=x_{1} v^{*}+u y_{1}^{*} .
$$

Similarly, we can see that there exist vectors $u_{1}, v_{1} \in K$ such that

$$
\phi\left(x w^{*}\right)=z_{1} v_{1}^{*}+u_{1} w_{1}^{*} .
$$

Standard arguments show that the last two relations imply existence of complex numbers $\alpha$ and $\beta$ such that

$$
\phi\left(x w^{*}\right)=\alpha x_{1} w_{1}^{*}+\beta z_{1} y_{1}^{*} .
$$

Similarly, there exist complex numbers $\gamma$ and $\delta$ such that

$$
\phi\left(z y^{*}\right)=\gamma x_{1} w_{1}^{*}+\delta z_{1} y_{1}^{*} .
$$

Let us now introduce operators $P=(1 / 2)(x-z)(y-w)^{*}$ and $N=$ $(x-z)(y+w)^{*}$. It is easy to verify that $P+\lambda N$ is an idempotent for every $\lambda \in \mathbb{C}$. As before, we see that there exists an integer $n \geq 2$ such that

$$
(\phi(P)+\lambda \phi(N))^{n}=\phi(P)+\lambda \phi(N)
$$

for every $\lambda \in \mathbb{C}$. The coefficient at $\lambda^{n}$ must be zero, and consequently,

$$
\phi(N)=\mu_{1} x_{1} y_{1}^{*}-\mu_{2} z_{1} w_{1}^{*}+(\alpha-\gamma) x_{1} w_{1}^{*}+(\beta-\delta) z_{1} y_{1}^{*}
$$

is a nilpotent operator. The linear span of the set $\left\{x_{1} y_{1}^{*}, z_{1} w_{1}^{*}, x_{1} w_{1}^{*}, z_{1} y_{1}^{*}\right\}$ is isomorphic to the set of all $2 \times 2$ matrices via the isomorphism

$$
a_{1} x_{1} y_{1}^{*}+a_{2} x_{1} w_{1}^{*}+a_{3} z_{1} y_{1}^{*}+a_{4} z_{1} w_{1}^{*} \mapsto\left\|\begin{array}{ll}
a_{1} & a_{2} \\
a_{3} & a_{4}
\end{array}\right\| .
$$

It is now easy to see that the fact that $\phi(N)$ is nilpotent is in a contradiction with $\mu_{1} \neq \mu_{2}$.

Thus, we have proved that $\phi(I)=c I$ for some root of unity $c$. The bijective linear mapping $\varphi: \mathscr{B}(H) \rightarrow \mathscr{B}(K)$ defined by $\varphi(A)=\bar{c} \phi(A)$ preserves potents in both directions. Moreover, we have that $\varphi(I)=I$. If $P$ is an arbitrary idempotent from $\mathscr{B}(H)$, then $P, I-P$, and $I-2 P$ are potent operators. The same must be true for $\phi(P), I-\phi(P)$, and $I-2 \phi(P)$. This implies that $\phi(P)$ is an idempotent. An application of Theorem 3 now concludes the proof of the implication (i) $\Rightarrow$ (iii).

Assume now that there exists an integer $r \geq 2$ such that $\phi$ preserves $r$-potent operators in both directions. As before we see that $\phi$ must be injective. Let $P, Q \in \mathscr{B}(H)$ be idempotents such that $P Q=Q P=0$. The same proof 
as in the finite-dimensional case [3] shows that $\phi(P) \phi(Q)=\phi(Q) \phi(P)=0$. Moreover, we have $\phi(P)^{r}=\phi(P)$ and $\phi(Q)^{r}=\phi(Q)$, and consequently,

$$
\phi\left((\lambda P+\mu Q)^{r}\right)=\lambda^{r} \phi(P)+\mu^{r} \phi(Q)=(\phi(\lambda P+\mu Q))^{r}
$$

for arbitrary $\lambda, \mu \in \mathbb{C}$.

Let $P$ be an idempotent from $\mathscr{B}(H)$. Then we have for every complex number $\lambda$ that

$$
\phi\left((P+\lambda I)^{r}\right)=\phi\left(((1+\lambda) P+\lambda(I-P))^{r}\right)=(\phi(P)+\lambda \phi(I))^{r} .
$$

Comparing the coefficients at $\lambda^{r-1}$ we obtain that

$$
r \phi(P)=\phi(P) S^{r-1}+S \phi(P) S^{r-2}+\cdots+S^{r-1} \phi(P),
$$

where $S=\phi(I)$. Multiplying this relation first from the left by $S$, then from the right by $S$, and using $S^{r}=S$ we get that $\phi(P) S=S \phi(P)$. Theorem 1 and surjectively of $\phi$ imply that $S$ belongs to the center of $\mathscr{B}(K)$. Applying $S^{r}=S$ once again, we conclude that $\phi(I)=c I$ for some $(r-1)$-root of unity.

The bijective linear mapping $\varphi: \mathscr{B}(H) \rightarrow \mathscr{B}(K)$ given by $\varphi(A)=\bar{c} \phi(A)$ preserves $r$-potent operators in both directions and satisfies $\varphi(I)=I$. We shall conclude the proof of implication (ii) $\Rightarrow$ (iii) and therefore the theorem by showing that it preserves idempotents. Let $P$ be an idempotent operator on $H$. Then we have $\varphi(P)^{r}=\varphi(P)$ and $(I-\varphi(P))^{r}=I-\varphi(P)$. Assume that $\sigma(\varphi(P))$ contains a complex number $\lambda \notin\{0,1\}$. From $|\lambda|=|1-\lambda|=1$ it follows that either $\lambda$ or $\bar{\lambda}$ is equal to $\exp ((1 / 3) \pi i)$, which is in contradiction with $\varphi(P)^{r}=$ $\varphi(P)$ in the case that 6 is not a divisor of $r-1$. In the case that 6 divides $r-1$ one can easily verify that $I+\exp ((2 / 3) \pi i) P$ and $I+\exp ((4 / 3) \pi i) P$ are $r$-potent operators. The same must be true for their $\varphi$-images. It follows that $\sigma(\varphi(P)) \subset\{0,1\}$, or in other words, $\varphi(P)$ is an idempotent. This completes the proof.

\section{REFERENCES}

1. P. Botta, S. Pierce, and W. Watkins, Linear transformations that preserve the nilpotent matrices, Pacific J. Math. 104 (1983), 39-46.

2. M. Brešar and P. Šemrl, Mappings which preserve idempotents, local automorphisms, and local derivations, Canad. J. Math. 45 (1993), 483-496.

3. __ Linear transformations preserving potent matrices, Proc. Amer. Math. Soc. 119 (1993), 81-86.

4. _ On local automorphisms and mappings that preserve idempotents, preprint.

5. M. D. Choi, D. Hadwin, E. Nordgren, H. Radjavi, and P. Rosenthal, On positive linear maps preserving invertibility, J. Funct. Anal. 59 (1984), 462-469.

6. M. D. Choi, A. A. Jafarian, and H. Radjavi, Linear maps preserving commutativity, Linear Algebra Appl. 87 (1987), 227-241.

7. J. Dieudonné, Sur une généralisation du groupe orthogonal à quatre variables, Arch. Math. 1 (1948), 282-287.

8. J. Dixon, Rigid embeddings of simple groups in the general linear group, Canad. J. Math. 29 (1977), 384-391.

9. A. A. Jafarian and A. R. Sourour, Spectrum-preserving linear maps, J. Funct. Anal. 66 (1986), 255-261.

10. N. J. Kalton, Sums of idempotents in Banach algebras, Canad. Math. Bull. 31 (1988), 448-451. 
11. D. R. Larson and A. R. Sourour, Local derivations and local automorphisms of $\mathscr{B}(X)$, Proc. Sympos. Pure Math., vol. 51, Part 2, Amer. Math. Soc., Providence, RI, 1990, pp. 187-194.

12. C. K. Li and N. K. Tsing, Linear preserver problems: A brief introduction and some special techniques, Linear Algebra Appl. 162-164 (1992), 217-235.

13. M. Omladič, On operators preserving commutativity, J. Funct. Anal. 66 (1986), 105-122.

14. __ On operators preserving numerical range, Linear Algebra Appl. 134 (1990), 31-51.

15. C. Pearcy and D. Topping, Sums of small numbers of idempotents, Michigan Math. J. 14 (1967), 453-465.

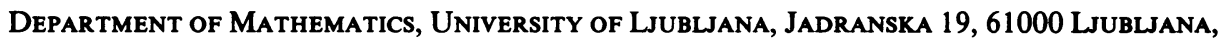
SLOVENIA

E-mail address: matjaz.omladic@uni-lj.si

E-mail address: peter.semrlouni-lj.si 